\title{
LOS CLUBES DE MIGRANTES EN SINALOA Y SUS POTENCIALIDADES DE DESARROLLO. ${ }^{1}$
}

Renato Pintor Sandoval ${ }^{2}$

Resumen. Como parte del desarrollo histórico de la migración mexicana a Estados Unidos, se han formado distintas organizaciones de migrantes por lugar de origen. Estas asociaciones han tenido un crecimiento considerable en las dos últimas décadas. La difusión de este fenómeno esquemático de organizaciones de migrantes ha sido últimamente trascendental para el aparato gubernamental en los niveles federal, estatal y municipal, por su capacidad e interés para actuar en las comunidades de origen del migrante en México. Sujetándose, a la experiencia zacatecana del 3x1 y convertido actualmente en una política nacional, el presente artículo analiza las potencialidades y debilidades de los clubes de oriundos que trabajan o no con dicha política en Sinaloa.

Palabras clave: migración sinaloense, clubes de migrantes, política nacional

Abstract. As part of the historical development of Mexican migration to the United States, they have formed various organizations of migrants by place of origin. These associations have had considerable growth over the past two decades. The spread of this schematic phenomenon of migrant organizations has recently been crucial for the government apparatus at the federal, state and municipal levels, their ability and interest to act in the communities of origin of migrants in Mexico. Clutching at the zacatecana $3 x 1$ experience and now become a national policy, this article analyzes the strengths and weaknesses of HTAs working or not with the policy in Sinaloa.

Keywords: sinaloense migration, HTA, $3 x 1$ national politics

\footnotetext{
${ }^{1}$ Este trabajo fue financiado con el recurso PROFAPI 2014/158 de la Universidad Autónoma de Sinaloa.

2 Doctor en Ciencias Políticas y Sociales con orientación en Relaciones Internacionales por la Universidad Nacional Autónoma de México. Profesor/investigador de la Facultad de Estudios Internacionales y Políticas Públicas de la Universidad Autónoma de Sinaloa. Miembro del CA de Redes Sociales y Construcción del Espacio Público. Miembro del SNI-I
} 


\section{Introducción:}

Actualmente, existen dos estrategias metodológicas para el abordaje del estudio de los clubes de origen de migrantes, una de ellas es la que postula la comprensión de la organización de los migrantes, de la cual Moctezuma (2011, 53), señala que se hacen juicios valorativos y rigurosos al aporte que realizan estas organizaciones en cuanto a sus inversiones sociales; y que se deshecha la distintas formas y al poder de interpretar dichas inversiones sociales, sin la naturaleza de la organización.

En el segundo grupo de investigadores, que miran la naturaleza de las inversiones, tales como García Zamora 2003) o González Gutiérrez (1999), vuelven a insistir en cómo aprovechar estos recursos del capital migrante en las zonas de expulsión, como la generación colectiva de empresas, servicios o como tanto se pregona, como una palanca de desarrollo regional. Debate que se centró en las remesas familiares primero, para posteriormente verlo a través de las remesas colectivas.

No es fortuito que se vuelva a discutir si el capital migrante puede transformar la estructura económica de sus lugares de destino; es por ello que desde los noventas se empezó por estudiar más concienzudamente a estos grupos de migrantes organizados por lugar de origen. La situación cobra importancia, tanto por el número de clubes, como por las obras que ellos realizan en pro de sus localidades de origen. Además, como parte del desarrollo de estas organizaciones o asociaciones, se ha puesto en marcha una política de acercamiento e incorporación del Estado mexicano hacia los migrantes, que intenta diseñar acciones y políticas públicas transnacionales (Pintor, 2015).

Es por ello, que las enriquecedoras experiencias de los estados de Zacatecas en 1986 y Guerrero en 1987, a través de sus programas 2x1, permitió que el gobierno de la República instaurara el programa 3x1 a nivel federal en 1992. Este trabajo analiza el programa 3x1 en Sinaloa, el cual se ha incrementado de 5 millones de pesos en 2002 a más de 15 millones en 2012.

A la par de dicho programa se analiza la participación de los Clubes de migrantes sinaloenses en inversiones comunitarias locales, los cuales están al margen de este programa, mostrándose como un recurso de alta calidad (Torres, 2001), que permite a los Clubes y Asociaciones de migrantes emprender inversiones sociales, fomentando 
así el capital social de las comunidades locales de los migrantes y sus prácticas transnacionales.

El primer apartado de nuestro trabajo, aborda brevemente la dinámica migratoria, no sólo internacional, sino nacional del estado de Sinaloa. En el segundo apartado, se analiza la participación de los clubes de origen sinaloense y sus potencialidades no sólo económicas sino sociales y culturales; y por último, en el tercer apartado, se analiza el programa $3 \times 1$ en Sinaloa, misma que algunos clubes rechazan este tipo de ayuda gubernamental, argumentando reglas de operación no tan favorables a su constitución como club de migrantes.

Aunque cabe destacar que el desarrollo de las redes sociales migrantes han ayudado a la creación de una asociación de organizaciones, que para el caso sinaloense es la Fraternidad Sinaloense en California.

\section{Sinaloa y sus migrantes.}

De acuerdo al informe del Consejo Nacional de Evaluación de la Política de Desarrollo Social, CONEVAL (2012), el estado de Sinaloa, guarda con respeto a las otras 32 entidades, el lugar 24 en población en pobreza y el 23 en porcentaje de población de pobreza extrema. Por lo que la entidad sinaloense, se ubica dentro de las 10 entidades con menor pobreza en el país.

Dicho informe nos arroja que el $36.5 \%$ de la población que habitaba en el estado se encontraba en situación de pobreza, con un promedio de 2.2 de 6 carencias $^{3}$, lo que representó 1, 011,013 de personas (CONEVAL, 2012). Asimismo, 5.4\% del total de la población sinaloense, se encontraba en situación de pobreza extrema, con un promedio de 3.6 carencias de 6 , o sea 150,172 personas, mientras que la pobreza moderada fue de $31.1 \%$ con un promedio de 2.0 carencias, es decir 860, 841 personas (CONEVAL, 2012).

Al desagregar la información a nivel municipal, vemos que existe ciertos municipios que presentan mayor porcentaje de población en pobreza, 11 de 18 municipios, destacándose Choix (79.1), Badiraguato (74.8), Cosalá (66.1), Sinaloa (63.0) y San Ignacio (61.3) (CONEVAL, 2012).

\footnotetext{
${ }^{3}$ Los indicadores son: rezago educativo, acceso a los servicios de salud, acceso a la seguridad social, calidad y espacios de viviendas, acceso a los servicios básicos en la vivienda y acceso a la alimentación.
} 
En resumen, en estos municipios más del $60 \%$ de la población se encontraba en situación de pobreza, lo que represento el $12.3 \%$ de la población del estado. Por otra parte los municipios que concentraron mayor número de personas en pobreza son: Culiacán 254, 056 (29.8\%), Ahome 136, 582 (30.6\%), Guasave 128, 744 (40.8\%) y Mazatlán 119, 926 (28.1\%) (CONEVAL, 2012).

Al realizar un análisis serio sobre la problemática del estado, la economía de Sinaloa en las tres últimas tres décadas aparece estancada en relación al avance promedio del resto de las entidades federativas de México (Soto, 2009, 246). En parte, se debe a que no existen las condiciones culturales empresariales.

Se encuentran empresas que se especializan en actividades intensivas de mano de obra barata, otras que tienen un alcance marginal, y otras que desfavorecen la aparición de empresas o empresarios, desde su expansión y diversificación, bajo nivel de oportunidades de nuevos mercados anclados históricamente en una cultura tradicional en el sector primario.

Además, Sinaloa es un estado cuyo motor económico se concentra en el sector agropecuario-exportador ${ }^{4}$ y las medidas de cambio estructural abarcaron numerosas medidas de liberalización comercial, entre las cuales se encuentra la eliminación de los precios de garantía de once cultivos considerados básicos.

Las reformas al agro también trajeron la desaparición de la Compañía Nacional de Subsistencias Populares (CONASUPO), la disminución de los subsidios a los insumos, la reducción del crédito y de los seguros y la reforma ejidal (Pintor, 2013, 253).

Si a esto le suma la presencia de una débil estructura industrial, una dependencia en el sector servicios cada vez menos competitivo y altos índices de inseguridad y violencia por el narcotráfico, se obtiene un estado potencialmente migrante (Sánchez S, 2010).

Estos cambios, propiciaron la existencia de densas redes migrantes locales rurales que se han entretejido tradicionalmente y que facilitan el éxodo regional y la existencia de una cultura migratoria arraigada, lo cual, aunado a problemas de

\footnotetext{
${ }^{4}$ La producción del sector rural se constituye, como el pilar de la economía del estado; sin embargo, hace ya tres décadas que este sector se ha visto inmerso en una fuerte crisis local, tanto de inversión, producción y de calidad.
} 
narcotráfico y reducida industrialización en la región, ha permitido que se reanime la migración en varias facetas y en circuitos migratorios tradicionales y nuevos (Sánchez S, 2014).

La entidad está conformada por migrantes rurales y periurbanos. Para ello, es necesario recurrir a información de las últimas décadas, tanto documentales, como trabajos de campo, buscando encontrar evidencias e interpretaciones de explicaciones del proceso migratorio sinaloense.

Sinaloa está inserto en un circuito transnacional migrante y, a la vez, en varios circuitos migratorios transestatales, entre ellos Durango, Oaxaca y Guerrero; por ejemplo, los sinaloenses se encuentran arraigados en ciudades fronterizas como Tijuana y Mexicali, B.C., que son los principales cruces de la frontera norte, según la EMIF ${ }^{5}$.

Aquí, los migrantes sinaloenses ocuparon el séptimo lugar con el 5.3\% del total nacional, en el 2005 (Lizárraga, 2005). De igual manera, datos del INEGI (2005), nos habla que el grueso de sinaloenses fuera del Estado, salieron de Sinaloa 101 mil 088 personas para radicar en otra entidad, tomado dicha información, dentro de los últimos 5 años, 28 de cada 100 se fueron a vivir a Baja California, Sonora, Baja California Sur, Jalisco y Nayarit.

Asimismo, el principal destino de la migración sinaloense internacionalmente, es el sur de California, principalmente, el área metropolitana de Los Ángeles, ${ }^{6}$ estimaciones realizadas por Ibarra (2005) señalan que la cantidad de sinaloenses en la región de Los Ángeles, ronda alrededor del 3\% del grueso de los migrantes mexicanos en el área.

Casi 450, 000 sinaloenses y un poco más de 650,000 personas de origen sinaloense habitan la región, conformada por gente proveniente de los municipios de Culiacán con 26,579 (33\%), Mazatlán con 9,658 (11.1\%), Mocorito con 7,395 (8.5\%),

\footnotetext{
${ }^{5}$ La EMIF es una encuesta que utiliza metodologías originales y se levanta en ocho localidades fronterizas del norte de México; aporta información sobre el número y características de los mexicanos que se trasladan a dichas localidades con la intención de permanecer en ellas o de cruzar a Estados Unidos para trabajar o buscar trabajo.

${ }^{6}$ Como la mayoría de los colonizadores de la Alta California vinieron de Sonora y Sinaloa, no es sorprendente que desde mediados del siglo XIX la gente se refiere a los Estados mencionados como la "madre patria". Otra importante característica, lo constituyen los patrones sociales y culturales básicos de la sociedad mexicana en alta California, los cuales se adoptaron en Sonora y Sinaloa, donde se había desarrollado durante los dos últimos siglos de colonización. (Castillo y Ríos, 1989, p. 39)
} 
Badiraguato con 5,829 (6.7\%), Guasave con 4,611 (5.3\%) y Ahome con 4,176 (4.8\%). Ver cuadro 1.

Cuadro 1.

Principales municipios migratorios sinaloenses

Ubicados en Los Ángeles, California (2003).

\begin{tabular}{|c|c|c|c|}
\hline Municipios & VSF & $\begin{array}{l}\text { Matrículas } \\
\text { consulares }\end{array}$ & Porcentaje \\
\hline \multicolumn{4}{|c|}{ TOTAL } \\
\hline Culiacán & 26,579 & 28,880 & 33 \\
\hline Mazatlán & 8,913 & 9,658 & 11,1 \\
\hline Mocorito & 6,825 & 7,395 & 8,5 \\
\hline Badiraguato & 5,380 & 5,829 & 6,7 \\
\hline Guasave & 4,255 & 4,611 & 5,3 \\
\hline Ahome & 3,854 & 4,176 & 4,8 \\
\hline El Rosario & 3,693 & 3,915 & 4,6 \\
\hline
\end{tabular}

Fuente: Ibarra (2005). Encuesta realizada en el Valle de San Fernando y estimaciones hechas con base en el registro de matrículas consulares.

Los municipios expulsores tradicionales son Badiraguato, Concordia, Cosalá, Choix, El Rosario y San Ignacio; los restantes se consideran como municipios de equilibrio, regiones que si lo comparamos con los datos del CONEVAL (2012), son los municipios que cuentan con un mayor número de pobreza en la entidad. Además, a esto hay que añadir, la investigación de Sánchez (2014), donde señala que aunque muchos de los que migran partiendo de zonas urbanas o de las ciudades sinaloenses, tienen como indicio que obedecen a redes rurales.

Sin embargo, los estudiosos de la migración, ponen a Sinaloa, como un estado en equilibrio o sin fuerte presencia de migración internacional, Pintor y Sánchez (2012), analizan que para determinar el grado migratorio de una región se debe considerar una serie de variables y elementos que pueden compartir y discernir con Estados o regiones lejanas o cercanas geográficamente.

Es claro que la ubicación en el país, es clave para determinar su posición regional, pero los elementos económicos y socioculturales determinan también su situación que permite determinar características similares, como ocurre con Sinaloa, atada a la región fronteriza de la migración (Durand y Massey, 2003). ${ }^{7}$

\footnotetext{
${ }^{7}$ Este tipo de zona concentra una migración que históricamente se ha presentado durante los últimos 50 años, a diferencia de la tradicional que lleva más 100 y la de reciente migración con 20 años. Además,
} 
Históricamente, Sinaloa ha sido un estado migrante. Los censos poblacionales de 1992 al 2010, muestran que 12.6\% del total de la población que reside en el Estado, entre 290 y 320 mil personas, habían cambiado de residencia municipal, en tanto que el índice nacional de este tipo de movimiento es de $16.5 \%$ de la población (INEGI, La migración en México, 2015: 245- 251). Mientras que en el Estado vivían entre 240 y 260 mil personas originarias de otras entidades federativas. En el Estado, sobresalen: Sonora (27.3\%), Baja California (20.5\%), Jalisco (19.8\%), Distrito Federal (12.4\%) y Chihuahua (4.4\%) (INEGI, 2015).

Así pues, el estado de Sinaloa incrementó su saldo migratorio de más de 370 mil para 1992 a casi los 500 mil sinaloenses que residen en el resto del país desde 2015 . Son dos estados los principales receptores de esa población: Baja California (44.2\%) y Sonora $(20.1 \%)$.

Esto significa que esas dos entidades fronterizas fueron el destino de cerca de dos terceras partes del total de los emigrantes (INEGI, 2015). Al considerar la migración exclusivamente a la frontera norte, por los principales cruce como Tijuana y Mexicali, datos del $\mathrm{EMIF}^{8}$, muestra que los migrantes sinaloenses ocuparon el séptimo lugar con el 5.3\% del total nacional (Lizárraga, 2005).

Dentro de los nuevos hallazgos sobre la dinámica migratoria sinaloense, las investigaciones de Montoya, García y Valenzuela (2008), encuentran un nuevo destino de migración sinaloense hacia Phoenix, Arizona: además sobresalen nuevos asentamientos como el norte de California o la zona de la Bahía de San Francisco, el estado de Washington, UTAH y Nevada. Recientemente, se han encontrado evidencias de asentamientos sinaloenses en la Unión Americana, como Carolina del Norte, Illinois y UTAH (Pintor, 2015).

En suma, Sinaloa es un estado de migrantes, que cuenta con un dinamismo propio y que más allá de los números de los censos, su situación es otra. El categorizar a Sinaloa en la región migratoria frontera-norte, puede considerarse como un riesgo, cuando se trata de hacer un análisis serio, ya que durante algún tiempo como una zona

Durand (2004) señala que en la región fronteriza han emigrado cerca de 20 millones de personas en 20 años, que es superada por los 40 millones de habitantes de la región central.

${ }^{8} \mathrm{La}$ EMIF es una encuesta que utiliza metodologías originales y se levanta en ocho localidades fronterizas del norte de México; aporta información sobre el número y características de los mexicanos que se trasladan a dichas localidades con la intención de permanecer en ellas o de cruzar a Estados Unidos para trabajar o buscar trabajo. 
regional con desarrollo económico considerable, funcionaba como paliativo a la migración de retención para muchos migrantes que iban hacia Estados Unidos.

\section{Los Clubes de migrantes sinaloenses.}

Partir del hecho de que cada región y cada pueblo de emigrantes son diferentes, nos lleva a entender la complejidad que se tiene en cuanto al estudio migratorio, por ejemplo, los estudios en general de migración internacional en México, son más numerosos en la zonas tradicionales de migración, especialmente como Zacatecas, Michoacán, Guanajuato y Jalisco, que los estudios de migración sobre Sinaloa (Ibarra, 2003 y 2007, García, 2003 y 2007, Montoya, 2004 y 2007, Lizárraga, 2005).

Dichos trabajos pertenecen a la llamada "región fronteriza de la migración", donde han sido aún poco documentados y resultan más escasos todavía los estudios sobre los clubes de migrantes sinaloenses (Pintor, 2016). Estos trabajos también consideran la dimensión sociocultural que subyace en tales iniciativas, que es un aspecto esencial para entender el nivel de desenvolvimiento político por el cual han transitado estas organizaciones.

Aunque los migrantes mexicanos, social y políticamente son distintos, ellos tienden a compartir actitudes específicas, iniciativas y están dispuestos a luchar por ellas. Buscan además, mantener unida la comunidad e implementar políticas públicas en sus regiones de origen. Al estudiar las redes que sostienen a los clubes de migrantes sinaloenses, vemos que así como algunas contribuyen de manera natural a aglutinar a los inmigrantes, también existen tendencias que promueven su desunión, sin embargo, esto no significa que pierdan su pertenencia e identidad comunitaria.

Se han documentado algunos casos de los clubes sinaloenses en California, el más antiguo, es el Club San José en Agua Verde, El Rosario, que data desde 1981, que a solicitud del párroco "Filemón", del poblado pidió se le construyera una iglesia nueva, misma que fue solventada gracias a la intervención Javier Benítez, líder del club de migrantes, proyectos que más tarde realizarían como la construcción de la Biblioteca Pública del poblado, ayuda en la escuela primaria, desayunador para niños, entre otras actividades más (Pintor, 2013, 220).

Siguiendo esa misma tesitura, la sindicatura de Chametla, localidad que pertenece al mismo municipio de El Rosario, fue formada en 1998, el "Grupo San 
Pedro", debe su nombre al santo patrono del lugar. Actualmente, cuenta con más de cincuenta integrantes cuya mayor parte radica en Los Ángeles, California.

Entre los apoyos otorgados por la asociación a su comunidad, destacan la construcción de un parque infantil, y las donaciones para la organización de las festividades por el aniversario del pueblo; asimismo, se organizan festejos para las personas de la tercera edad. Los miembros del club participan también en la donación de juguetes en Navidad y han apoyado con material de papelería y equipos de cómputo a las escuelas de su comunidad (RFS, 2000).

Otro grupo, lo componen los oriundos de la cabecera municipal de El Rosario, radicados también en Los Ángeles, California, y que aglutina aproximadamente a 90 integrantes. El club se formó gracias a la tenacidad de Concepción Aragón de Coob. Dicha organización empieza a apoyar a su comunidad de origen en el trienio 1986-88, en la administración municipal de Luis Villegas.

Una de las primeras acciones de esta organización fue la adquisición y remodelación de un edificio para la conformación del asilo de ancianos, que representó un gasto de aproximadamente 400 mil pesos (García y Pintor, 2008). Desde entonces y hasta la actualidad, esa noble institución continúa sus funciones y los gastos de operación corren a cargo del club.

También destaca el "Club Bamoa" formado por migrantes residentes en California. Los oriundos de esta sindicatura perteneciente al municipio de Guasave, Sinaloa recaudan fondos por medio de diferentes tipos de actividades, como cenas, rifas y viajes, para pavimentar calles y banquetas de su localidad (Pintor y García, 2008).

Ahora bien, no todos responden de la misma manera, a pesar de la masividad y las asociaciones de migrantes. Cuando la influencia cultural es alta, es porque en sus lugares de origen, prevalece una cultura migratoria sumada a la existencia de una o varias asociaciones de migrantes, y al gran número de personas que tienen documentos para realizar viajes, lo cual permite el desarrollo de negociaciones con las comunidades y los gobiernos locales.

Esta influencia del grupo de migrantes, puede traducirse en demandas en los proyectos sociales (Imaz, 2003).

Moctezuma (2003, 149-152 y 2011, 58) sostiene que el estudio de las redes sociales migrantes no deben de detenerse o limitarse sólo a la aparición del club de 
migrantes, sino que su estudio debe ampliarse hacia la conformación de confederaciones o agrupaciones de clubes de migrantes:

Una vez que se ha consolidado la vida comunitaria, el liderazgo de los migrantes hace emerger las primeras manifestaciones organizativas, las cuales, al contar con el estímulo del Estado y particularmente de los gobernantes por entidad o municipio, es posible que esa organización vaya más lejos.

(Moctezuma, 2003: 149-152 y 2011, 58).

Existen casos donde varias comunidades filiales se unen y forman un solo club social, pero hay clubes con autonomía, en los que cada miembro reconoce un origen distinto pero con un objetivo común. Empero, aunque los clubes de migrantes derivan de las comunidades filiales, es importante acotar su diferencia.

Lo más común en los estudios del transnacionalismo es encontrar una organización simple que emprende obras en su comunidad de origen, pero no se pierde de vista que un club es parte de una asociación y que sin ella no tendría capacidad de negociación frente al estado o municipio (Moctezuma, 2003).

En el caso de los migrantes sinaloenses en California, su federación se encuentra agrupada en la Fraternidad Sinaloense en California (FSC) que fue fundada en 1991, y cuya misión radica en organizar a los catorce clubes de oriundos californianos registrados hasta el 2009, con bailes de coronación de reinas, así como suscribir convenios con sus respectivas autoridades estatales (Revista de la Fraternidad Sinaloense, 1998, 1998 a y García y Pintor, 2008), las cuales se tendrían que comprometer a secundar el trabajo social que llevan a cabo los clubes en las comunidades nativas (Véase el cuadro 2).

La Fraternidad Sinaloense en California, aglutina a 11 clubes de migrantes, por lo que la Asociación ha buscado un nuevo dinamismo en los trabajos de buscar una fuente de financiación alterno al gobierno estatal de Sinaloa y al consolidarse como una organización no lucrativa, bajo la presidencia de José Ángel Barajas Patrón.

La Fraternidad Sinaloense en California ha sostenido y trata de incentivar la participación en el programa $3 \times 1$, aunque, en ocasiones lo prefieren hacer fuera del $3 \times 1$, pero si tratan de tener correspondencia con las acciones de políticas estatales, municipales e incluso federales. 
Vol. 7, No. 12, 2017.

\begin{tabular}{|c|c|c|c|c|c|}
\hline \multicolumn{6}{|c|}{$\begin{array}{l}\text { CUADRO 2. CLUBES SINALOENSES INSCRITOS EN LA FRATERNIDAD SINALOENSE Y SUS } \\
\text { PRINCIPALES OBRAS SOCIALES }(2010)^{9}\end{array}$} \\
\hline Nombre del club & $\begin{array}{c}\text { Año de } \\
\text { Fundación }\end{array}$ & Presidente & $\begin{array}{l}\text { Frecuencia } \\
\text { de reunión }\end{array}$ & $\begin{array}{l}\text { Proyectos } \\
\text { principales }\end{array}$ & $\begin{array}{l}\text { Miembros } \\
\text { activos }\end{array}$ \\
\hline San Ignacio & 1993 & Adriana Noriega & & & \\
\hline Guasave & 1992 & Ramón Cárdenas & $\begin{array}{c}\text { Cada } 2 \\
\text { meses }\end{array}$ & $\begin{array}{l}\text { Apoyo comunitario } \\
\text { y entrega de carro de } \\
\text { bomberos }\end{array}$ & 25 \\
\hline Rosario & 1985 & Octavio Solorza & Cada mes & $\begin{array}{l}\text { Rec. del asilo y } \\
\text { apoyos a la } \\
\text { comunidad }\end{array}$ & 20 \\
\hline Escuinapa & 1991 & Lourdes Arroyo & Cada mes & Jardín de niños & 25 \\
\hline Agua Verde & 1981 & Javier Benítez & Cada mes & $\begin{array}{l}\text { Construcción de } \\
\text { iglesia y biblioteca }\end{array}$ & 25 \\
\hline El Roble & 1992 & José Ibarra & $\begin{array}{c}\text { Cada } 2 \\
\text { meses }\end{array}$ & $\begin{array}{c}\text { Apoyos } \\
\text { comunitarios }\end{array}$ & 6 \\
\hline Los Pericos & 1993 & Leticia Camacho & Cada mes & & \\
\hline Mazatlán & 1993 & Octavio Barrón & Cada mes & $\begin{array}{c}\text { Desayunos } \\
\text { escolares, sistema de } \\
\text { alumbrado en } \\
\text { colonias necesitadas }\end{array}$ & 50 \\
\hline Ahome & 1993 & José Zarate & Cada mes & $\begin{array}{l}\text { Envío de } 50 \text { sillas de } \\
\text { ruedas }\end{array}$ & 14 \\
\hline El Fuerte & 1993 & $\begin{array}{l}\text { Ma. del Refugio } \\
\text { Reyna }\end{array}$ & Cada mes & Carro de bomberos & 14 \\
\hline Culiacán & 1993 & Antonio Malacón & Cada mes & $\begin{array}{l}\text { Carro de bomberos y } \\
\text { remodelación de la } \\
\text { plazuela }\end{array}$ & 25 \\
\hline
\end{tabular}

Fuente: Revista de la Fraternidad Sinaloense, Los Ángeles, julio de 1998 y $1998^{\mathrm{a}}$.

Desde su establecimiento, la fraternidad Sinaloense en California, a instancias del entonces Cónsul José Ángel Pescador Osuna, de origen mazatleco y por el guasavense Humberto Gálvez y el aguaverdense Javier Benítez (Pintor, 2013, 222), la FSC, ha tenido como objetivo la de representar a la comunidad migrante ante las autoridades tanto en México como en California. En palabras de su actual presidente, José Ángel Barajas, la Fraternidad se visualiza como:

\footnotetext{
${ }^{9}$ El Club Cubiri, el Club Caiteme, el Club Costa Rica y el Club Rosa Morada (Mocorito) adquirieron su adscripción a la Fraternidad en el lapso de 2008-9, y sus inicios fueron apoyados por el IME para su formación y adscripción a la Fraternidad Sinaloense en Los Ángeles.
} 
Una fuerte organización no lucrativa que cuenta con oficina y centro de negocios, establecida en varias regiones de California; con una imagen sólida frente a la comunidad, con un desarrollo económico estable, así como con un incremento continuo en la adhesión de nuevas comunidades de sinaloenses en California, lo cual ha permitido forjar nuevos líderes.

Entrevista con José Ángel Barajas Presidente de la Fraternidad Sinaloense en California 16 de mayo de 2011.

La fraternidad, hoy en día, cuenta con el apoyo de SEDESOL por medio del programa $3 \times 1$, para lograr objetivos de provecho a la comunidad, realizando proyectos de interés comunitario como carreteras, pavimentación de calles, electrificación, remodelación de iglesias, escuelas, etc. (RFS, 2008). Los diferentes líderes de las comunidades y el aval de la Fraternidad, permiten nuevas estrategias para mejores proyectos que busquen el desarrollo económico de Sinaloa y California (Pintor, 2013, 225, ,

Sumándose a proyectos socioeconómicos, la Fraternidad conjuntamente con el gobierno del estado, participó en el proyectos 'Rescate de la Sierra', que están amparados también bajo el proyecto $3 \mathrm{x} 1$, y que tienen como objetivo principal que ciertas comunidades de la sierra puedan contar con plantas de luz y de agua, así como de piso de cemento y servicio médico, a través de brigadas, caminos rurales, etc.

Actualmente se encuentran realizando esfuerzos por recaudar fondos que serán donados para el proyecto de la Universidad del Valle del Évora en el Estado de Sinaloa (RFS, Informe de labores, 2008).

No sólo a nivel rural destacan los clubes sinaloenses, también lo hacen los clubes de migrantes de origen urbano, como el "Club Mazatlán”, que trabaja con sus residentes de Los Ángeles, ayudando a niños y gente de la tercera edad que están en los orfanatos, la Casa Hogar y en la cárcel municipal del puerto.

Apoyan también con desayunos escolares en comunidades de las zonas rurales del municipio y en la organización de eventos deportivos entre equipos residentes en Los Ángeles y Mazatlán (Pintor y García, 2008). 


\section{El programa 3x1 en Sinaloa.}

Partiendo de la experiencia del Programa " $2 \mathrm{X} 1$ " en Zacatecas y posteriormente el 3x1 a nivel Federal, acciones que permiten anticipar lo importante de la organización desde la perspectiva de las comunidades migrantes transnacionalizadas que ayudan a realizar obras públicas en sus comunidades de origen, con el ahorro social enviado desde los Estados Unidos.

Actualmente el programa de SEDESOL 3x1, ha ganado la aceptación de líderes de organizaciones de migrantes y de líderes locales. Cuenta con oficinas en California e Illinois y en 20154 el programa operó en 23 estados de la República, atendió a cerca de 900 grupos asentados en 31 estados de la Unión Americana e incluyo asesoría y capacitación (Imaz, 2007, 205).

El Programa con casi quince años de funcionamiento deja varias lecciones, mismas que han sido retomadas en el estado de Sinaloa. Pero antes, es importante señalar, que la creación del Ramo 33 en 1997, fue un parteaguas para el fortalecimiento de los municipios, como un actor participante en el proceso de conformación del 3x1 (Soto, 2006, 223 y Valenzuela 2006 ${ }^{\text {a }}$ 145).

Entre los rubros principales de este ramo y el 3x1, es la construcción de infraestructura que a su vez, a través de SEDESOL y el Programa Empleo Temporal (PET), va a permitir "generar oportunidades de ingreso en el medio rural con acciones dirigidas al desarrollo de capital humano que vive en pobreza y pobreza extrema" (SEDESOL, pág. electrónica, 2014 y PND, 2007).

En Sinaloa, al igual que otras entidades, el programa opera a través del Departamento de la Dirección de Apoyo a Empresas Sociales de SEDESOL, destacándose, la construcción de obras de infraestructura básica y el monto total ha sido de 69, 108,918, en diez años, siendo la aportación federal de 32, 145,681, y la de los clubes similar a dicha aportación, como se ve en el siguiente Cuadro 3. 
Vol. 7, No. 12, 2017.

Cuadro 3. El programa 3x1 en Sinaloa y su distribución de ingreso, 2002-2012

\begin{tabular}{|c|c|c|c|c|c|}
\hline Año & $\begin{array}{c}\text { Inversión } \\
\text { Total }\end{array}$ & Gobierno Federal & $\begin{array}{c}\text { Gobierno } \\
\text { Estatal }\end{array}$ & $\begin{array}{c}\text { Gobierno } \\
\text { Municipal }\end{array}$ & $\begin{array}{c}\text { Clubes de } \\
\text { Migrantes }\end{array}$ \\
\hline $\mathbf{2 0 1 2}$ & $15,503,140$ & $7,344,570$ & S/D & $2,829,288$ & $5,329,285$ \\
\hline $\mathbf{2 0 1 1}$ & $22,103,230$ & $8,100,944$ & $3,992,195$ & $2,947,402$ & $7,062,687$ \\
\hline $\mathbf{2 0 1 0}$ & $8,522,395$ & $4,261,197$ & S/D & S/D & $4,261,197$ \\
\hline $\mathbf{2 0 0 9}$ & $4,935,943$ & $2,467,971$ & S/D & S/D & $2,467,971$ \\
\hline $\mathbf{2 0 0 8}$ & $8,291,414$ & $4,145,707$ & S/D & S/D & $4,145,707$ \\
\hline $\mathbf{2 0 0 7}$ & $4,280,455$ & $2,140,227$ & S/D & S/D & $2,140,227$ \\
\hline $\mathbf{2 0 0 6}$ & $3,061,121$ & $1,530,560$ & S/D & S/D & $1,530,560$ \\
\hline $\mathbf{2 0 0 5}$ & $1,438,000$ & 719,000 & S/D & S/D & 719,000 \\
\hline $\mathbf{2 0 0 4}$ & S/D & S/D & S/D & S/D & S/D \\
\hline $\mathbf{2 0 0 3}$ & $3,757,595$ & S/D & S/D & S/D & S/D \\
\hline $\mathbf{2 0 0 2}$ & $5,738,020$ & $1,435,505$ & S/D & S/D & $1,435,505$ \\
\hline
\end{tabular}

Esta tabla nos indica, que el peso del programa recae principalmente en las aportaciones que realizan el gobierno Federal y los Clubes de migrantes, con jorobas importantes, pero también revela que la capacidad de los clubes de migrantes sinaloenses, en ocasiones está por encima de las autoridades estatales y locales.

Recientemente, desde los 80's, como argumenta Pintor y Sánchez, (2014), el gobierno mexicano ha seguido una política de institucionalización y de acercamiento a través de diferentes políticas que contemplan al Estado mexicano con su diáspora, estas han pasado de un periodo de indiferencia política presente desde las décadas de los sesenta, hasta finales de los ochenta a una relación de cooptación y displicencia marcada por un trato corporativista y clientelar (Valenzuela, 2006).

Mientras que para los estados, han existido infinidad de ejemplos, donde se da constancia de reuniones de los gobernadores y sus migrantes, para el de Sinaloa; sin embargo, existe aún una nula política entre el gobernador y las comunidades de emigrados; se describe, por un lado, como una relación "cómoda", “orgánica," o "funcional", mientras que por el otro, "superficial" e "intrascendente", principalmente, partiendo del hecho de que la relación entre los migrantes organizados y el gobernador de un estado es de una doble naturaleza en el sentido de que al igual que se hace de fuertes reclamos y críticas, también está hecha de peticiones y demandas de inclusión no sólo a las tareas que le son propias al gobierno (Valenzuela, 2006). 
De igual manera, el cuadro nos presenta que el diseño del programa $3 \times 1$ en Sinaloa, tienen como sostén y respaldo a las organizaciones de migrantes, en particular los clubes de paisanos (oriundos), principalmente, a los que ya se encuentran organizados y no a la promoción de organizarse.

Entre los municipios que más han visto beneficiados con este programa, son Guasave (40), Ahome (23), Choix (15), Sinaloa (17), Culiacán (17), San Ignacio (8) y Elota (18) (Sedesol-Sinaloa, 2010). Este último, ha llamado la atención, ya que aglutina cerca de la tercera parte de los fondos de los 89 obras completadas y que para el ejercicio 2014, esperan finalizar con 175, que se han realizado en diez años bajo el programa 3x1, pero que a su vez es constante, (Sedesol-Sinaloa, 2010), como se aprecia en el siguiente cuadro 4.

Cuadro 4. Obras realizadas con el programa 3x1 en Sinaloa, por municipio, 2002, 2012.

\begin{tabular}{|c|c|c|c|c|c|c|c|c|c|c|c|c|}
\hline Municipios & $\mathbf{2 0 1 2}$ & $\mathbf{2 0 1 1}$ & $\mathbf{2 0 1 0}$ & $\mathbf{2 0 0 9}$ & $\mathbf{2 0 0 8}$ & $\mathbf{2 0 0 7}$ & $\mathbf{2 0 0 6}$ & $\mathbf{2 0 0 5}$ & $\mathbf{2 0 0 4}$ & $\mathbf{2 0 0 3}$ & $\mathbf{2 0 0 2}$ & Total de obras \\
\hline Ahome & 2 & 11 & 3 & - & 5 & - & - & - & - & 1 & 1 & 23 \\
\hline Angostura & - & - & - & 1 & 1 & - & - & - & - & - & 1 & 3 \\
\hline Badiraguato & - & - & - & - & 3 & - & - & - & - & - & - & 3 \\
\hline Choix & 3 & 5 & 2 & 2 & 2 & 1 & - & - & - & - & - & 15 \\
\hline Concordia & - & - & - & - & - & - & - & 1 & - & - & - & 1 \\
\hline Cosalá & - & - & - & - & - & - & - & - & - & - & - & 0 \\
\hline Culiacán & 4 & 5 & 1 & 1 & - & - & - & - & - & - & 6 & 17 \\
\hline El Fuerte & 1 & - & - & - & - & - & 1 & 1 & - & 2 & 2 & 7 \\
\hline Elota & 1 & 1 & 3 & 2 & 1 & 6 & 1 & 1 & - & 1 & 1 & 18 \\
\hline Escuinapa & - & - & - & - & - & - & - & - & - & 2 & 1 & 3 \\
\hline Guasave & 2 & 9 & 5 & 7 & 1 & 5 & 5 & - & - & 1 & 5 & 40 \\
\hline Mazatlán & - & - & - & 1 & - & - & - & - & - & - & - & 1 \\
\hline Mocorito & - & - & - & & 1 & - & - & 1 & - & - & 3 & 5 \\
\hline Navolato & 1 & - & - & - & - & - & - & - & - & - & 2 & 3 \\
\hline Rosario & - & - & - & - & - & 5 & 2 & 1 & - & - & - & 8 \\
\hline S. Alvarado & - & - & - & - & - & - & - & - & - & 2 & 1 & 3 \\
\hline San Ignacio & - & - & - & - & - & 2 & 2 & - & - & 4 & - & 8 \\
\hline Sinaloa de L & - & - & 6 & - & 2 & 1 & 1 & 1 & - & 5 & 1 & 17 \\
\hline Obras por año & $\mathbf{1 4}$ & $\mathbf{3 1}$ & $\mathbf{2 0}$ & $\mathbf{1 4}$ & $\mathbf{1 6}$ & $\mathbf{2 0}$ & $\mathbf{1 2}$ & $\mathbf{6}$ & $\mathbf{0}$ & $\mathbf{1 8}$ & $\mathbf{2 4}$ \\
\hline
\end{tabular}

Fuente: Elaboración propia con datos de SEDESOL, 2014.

Además, este cuadro nos muestra que los municipios con menor número de obras construidas son, Angostura, Badiraguato, Escuinapa, Navolato y Salvador Alvarado con 3 obras cada uno, seguido de Mazatlán y Concordia con una sola obra y Cosalá, el municipio con más alto nivel de pobreza y en situación extrema, según el 
CONEVAL, 2012, con ninguna obra realizada. Dando esto como resultado que en el estado de Sinaloa en promedio se construyen casi 18 obras con 5.75 millones de pesos anualmente.

Para el gobierno de Sinaloa, actualmente, son los municipios los espacios donde "pilotean", ensayan, nuevas formas de tratar la migración a través de programas -sean adaptaciones federales o diseños propios. Para muchos presidentes municipales, los programas existentes, y la búsqueda de la aplicación de los programas a través de los clubes de oriundos permiten crear espacios transnacionales sólidos, como lo argumenta Guarnizo (2003), en donde sostiene que "la participación del Estado es sumamente importante, ya que pueda recrear espacio trasnacionales".

O bien, siguiendo esa misma lógica de Guarnizo (2003), nos habla que "la vida transnacional no ha representado obstáculo alguno para que los inmigrantes participen en el ámbito político de sus comunidades de origen"; sino todo lo contrario, "ha permitido el surgimiento de nuevos actores sociales que influyen en los procesos políticos de sus comunidades", dando lugar a la apertura de arenas políticas de negociación social entre sus gobiernos.

Uno de estos casos es la Fraternidad Elotense en California, que gracias al programa $3 \times 1$, dieron pie a que se construyera una secundaria técnica, electrificación y alcantarillado en ese municipio. Esto se debe en parte a un trabajo en conjunto entre el alcalde Rodríguez Castillo y líder del club Radamés Díaz Meza, que además ayudaron a que el municipio fuera incluido en el Programa "Cruzada Nacional contra el hambre" en el 2014 (El debate, marzo 2014).

Dicho Club, a través del 3x1, investigación realizada por Villegas $(2014,87)$, encuentra que durante el periodo 2002-2013, se han llevado a cabo 18 obras en el municipio, lo que representa el $10.45 \%$ del total estatal. Éstas tienen un valor de inversión que asciende a más de 20 millones de pesos, cantidad que constituye el 16.51 $\%$ de todas las inversiones realizadas en Sinaloa. También, al desagregar las aportaciones realizadas, encontramos que el gobierno de Elota es el que más contribuciones monetarias ha realizado, con el $23.17 \%$ del total.

En general, los programas más demandados, bajo el programa 3x1 en Sinaloa, son espacios deportivos (68), mejoramiento urbano, principal actividad en el 2006 
(recarpetado, 46), electrificación (15), centros de salud (9), Productivo Bifamiliar y productivo unifamiliar (9) (Sedesol-Sinaloa, 2014), entre otras más, como se aprecia en la siguiente Cuadro 5:

\begin{tabular}{|c|c|c|c|c|c|c|c|c|c|c|c|c|}
\hline \multicolumn{12}{|c|}{ Cuadro 5. Tipos de obras realizadas bajo el programa 3x1 en Sinaloa, 2002-2012. } & \multirow[b]{2}{*}{$\begin{array}{c}\text { Total de } \\
\text { obras }\end{array}$} \\
\hline Tipo de obra & 2012 & 2011 & 2010 & 2009 & 2008 & 2007 & 2006 & 2005 & 2004 & 2003 & 2002 & \\
\hline Centro de Salud & 2 & 2 & 1 & 1 & 1 & - & - & - & - & 1 & 1 & 9 \\
\hline Espacios deportivos & 6 & 21 & 5 & 9 & - & 5 & 5 & - & - & 4 & 13 & 68 \\
\hline Productivo Bifamiliar & 5 & 4 & - & - & - & - & - & - & - & - & - & 9 \\
\hline $\begin{array}{l}\text { Productivo } \\
\text { unifamiliar }\end{array}$ & - & 4 & 5 & - & - & - & - & - & - & - & - & 9 \\
\hline $\begin{array}{c}\text { Equipamiento } \\
\text { Municipal }\end{array}$ & 1 & - & - & 1 & 1 & - & - & - & - & 1 & - & 4 \\
\hline Áreas Culturales & - & - & 1 & - & 3 & - & - & - & - & - & - & 4 \\
\hline $\begin{array}{c}\text { Mejoramiento } \\
\text { Urbano }\end{array}$ & - & - & 7 & 3 & 4 & 10 & 6 & 6 & - & 9 & 1 & 46 \\
\hline Productivo & - & - & 1 & - & - & - & - & - & - & - & - & 1 \\
\hline Centros educativos & - & - & - & - & 3 & 1 & 1 & - & - & 1 & & 6 \\
\hline Electrificación & - & - & - & - & 4 & 4 & - & - & - & & 7 & 15 \\
\hline Agua Potable & - & - & - & - & - & - & - & - & - & 2 & 1 & 3 \\
\hline Drenaje & - & - & - & - & - & - & - & - & - & - & 1 & 1 \\
\hline Total por año & 14 & 31 & 20 & 14 & 16 & 20 & 12 & 6 & 0 & 18 & 24 & 175 \\
\hline
\end{tabular}

Fuente: Elaboración propia con datos de

Sedesol Sinaloa,2014.

Sin embargo, aún que se evidencia el apoyo entre los clubes de migrantes y las autoridades, estas responden a necesidades presupuestales del municipio, pero también a problemas más estructurales como son la dependencia de las economías locales y el despoblamiento (Valenzuela, 2006).

En sí, la participación de las autoridades locales y los clubes de migrantes no son autónomas, estas se ajustan, principalmente a las reglas de operación del programa 3x1, y a las diversas formas de negociar su aplicación, se dice que los migrantes sólo sugieren las obras a realizar y que es el municipio (un comité del gobierno municipal compuesto por el Consejo de Planeación Municipal y miembros del cabildo, entre otros) el que da prioridad y determina cuáles son las más importantes.

Sin embargo, en la práctica, son varios los casos en que los clubes de oriundos negocian con el municipio el apoyo de una obra a cambio de conceder la firma de otra en la que el gobierno municipal tiene interés real en llevar a cabo, aunque también en el transcurso de las obras puede llevar a cabo problemas, y no realizar ninguna, como se ve en el siguiente Cuadro 6. 


\section{Cuadro 6. Beneficios y Problemática del Programa 3x1}

\section{Beneficios}

*Su enfoque es de filantropía social.

*Responde a motivaciones humanitarias y de prestigio social (1)

*Pueden crear proyectos empresariales.

*El Migrante y la sociedad civil son activos promotores de los programas.

*Buena aceptación entre comunidades y entre los migrantes (1)

* Sirven para recrear y fomentar las acciones sociales de una sociedad

\section{Problemática}

* Su base son las Remesas colectivas por lo que dependen de la organización y de los fondos de los migrantes.

*La responsabilidad de la ejecución del proyecto queda en gobiernos locales.

*Aprobada la obra, los clubes y beneficiarios locales no participan en el seguimiento (1)

* La capacidad de promoción y captación de fondos de los clubes ha rebasado la capacidad presupuestal de los gobiernos locales (1)

* No se hacen previsiones para su mantenimiento posterior.

*Exceso de burocratismo

Fuente: Pintor, (2011), El habitus y los campos transnacionales en el proceso del transnacionalismo migrante en Migraciones Internacionales, vol.6 no.2 México jul. /dic.

La gran problemática, es la "decisión” de las obras a construir, como se menciona, si estas obedecen a la autonomía o no, ya que no todos los presidentes municipales de pueblos de migrantes están dispuestos a aceptar esta situación y se dice que los escuchan, comentan con ellos, dialogan, negocian, sobre la pertinencia de construir una obra, pues "Lo que a veces ellos quieren a nosotros no nos interesa; entonces hay que negociar, convencerlos, venderles la idea de que nos apoyen. Motivarlos a que inviertan en su municipio, por lo que muchos clubes y líderes de éstos realicen inversiones al margen del presupuesto del programa.

En sí, los presidentes municipales saben que la decisión sobre las obras a financiar es un asunto que debe resolverse basado en el principio de la "sociedad" entre partes involucradas. Bajo este principio, es entonces que se llega a reconocer que los migrantes organizados sí deciden pero también el municipio.

\section{Conclusiones}

En términos generales, la entidad carece o más bien, ignora el problema de la migración, principalmente, el de partida, en este sentido urge la necesidad de crear mecanismos e instituciones de acercamiento, como en la de otros estados. Por ejemplo, 
Sinaloa es uno de los pocos estados, donde 28 de los 32 , que no cuenta con un instituto u oficina de atención de sus comunidades de emigrados.

Dicho instituto, permitiría tener una política estatal de migración, que no sólo debería de incluir los arreglos de los espacios públicos de los migrantes, entiéndase la de sólo incluirlos en la realización de proyectos de coparticipación, sino la de mejorar sus vidas, como el de asesoría legal, financiera, familiar, comercial, entre otras funciones.

De igual manera, Alba (2010), nos dice que distintos gobiernos han sugerido llevar la dimensión migratoria y sus impactos económicos a políticas económicas a través de programas, en donde se le ha llamado por migratizar las políticas de desarrollo, en donde el gobierno asume las bondades de las remesas colectivas y principalmente las familiares.

Sin embargo, un punto a destacar de las remesas colectivas y la organización migrante es la escasa transferencia relativa de recursos. ${ }^{10}$ Sólo una mínima parte del total de las remesas familiares son transferibles por organizaciones de migrantes que envían dinero a sus comunidades para apoyar la construcción de infraestructura pública y el desarrollo de proyectos sociales.

No obstante, las dificultades y los tropiezos a los que han de enfrentarse las organizaciones de migrantes y sus proyectos sociales han de apreciarse como óptimos, pues el impacto social en la comunidad de origen es precisamente uno de los objetivos primordiales de los clubes de migrantes y de la sociedad local, porque "tratan de no ser excluidos" (Sánchez, García y Pintor, 2015), sino de alcanzar una “integración social”.

Finalmente, al detallar sobre la operación del programa 3x1 en Sinaloa, podemos ver que existen dos polos opuestos, una donde los gobiernos locales influyen en sus filiales en Estados Unidos, tratando de orientarlos para que sus proyectos sociales en la entidad se cristalicen, y por el otro, existe para algunos presidentes municipales sinaloenses una idea vaga sobre los aportes que puede generar la organización de

\footnotetext{
${ }^{10}$ En sus estudios sobre este tipo de recursos, Torres, comenta que la organización, las formas y el empuje que tienen estas organizaciones son recursos que bien pueden ser de alta calidad Torres, (2001).
} 
migrantes, ya sea con o sin el apoyo del programa, por lo que el uso de este capital social y organizativo puede generar recursos de alta calidad, debido al diseño, organización e implementación de las inversiones sociales en las localidades de los migrantes. 


\section{Referencias}

(2007), Vidas Compartidas. Formación de una red migratoria transnacional, de Aguacaliente, Sinaloa a Victor Valley, California". México: Plaza y Valdez. México.

(2007). Encuesta a Hogares Sinaloenses sobre Migración y Desarrollo Social en Sinaloa, 2006, Centro de Estudios sobre la globalización y el Desarrollo Económico (CEGDER), Facultad de Estudios Internacionales y Políticas Públicas, UAS, Sinaloa (resultados preliminares).

(2011). La transnacionalidad de los sujetos. Dimensiones, metodologías y prácticas convergentes de los migrantes mexicanos en Estados Unidos. Miguel Ángel Porrúa y Universidad Autónoma de Zacatecas, México.

(2013). "Las inversiones sociales en Agua Verde y el desgaste del proceso transnacional" en Ismael García Castro y Alex Munguía Salazar (Cords). Universidad Autónoma de Sinaloa y Jorale Editores. Sinaloa.

Alba, F. (2010). "Extendiendo el alcance de las políticas públicas en materia migratoria" en Paula Leite y Silvia E. Giorguli (Cord), Reflexiones a la emigración mexicana, CONAPO, México.

Consejo Nacional de Evaluación de la Política de Desarrollo Social (2012). Informe de pobreza y evaluación en el estado de Sinaloa 2012. México, D.F. CONEVAL.

Durand, J. y Douglas S. (2003).Clandestinos: Migraciones, México- Estados Unidos en los albores del siglo XXI, CIESAS-U de G., México.

García, I. (2003). Confeccionando desilusiones, DIFOCUR y el Centro de Estudios de la Globalización y el Desarrollo Regional de la Universidad Autónoma de Sinaloa.

García, R. (2003). "Los Proyectos Productivos con los emigrantes en México, Hoy” en Revista Arenas, UAS, núm. 5, octubre- diciembre.

González, C. (2006). "Del acercamiento a la inclusión institucional: la experiencia del Instituto de los Mexicanos en el Exterior en Carlos González Gutiérrez (Cords). Relaciones Estado Diáspora: aproximaciones desde cuatro continentes. Miguel Ángel Porrúa-SRE, México

González, C. (1999). "Promoviendo Identidades: Las Relaciones del Estado Mexicano con las Comunidades de Origen Mexicano en Estados Unidos" en Gail Mummert (ed.), Fronteras Fragmentadas, COLMICH, México.

Ibarra, G. (2003). La población sinaloense en Los Ángeles en el contexto de la migración mexicana a Estados Unidos. Origen municipal, distribución territorial y pobreza, en el "Segundo Encuentro de Emigración Sinaloense en el Extranjero: Ciudadanía, Identidad y Participación Político Electoral”, Lynwood, California, 24-27 de abril de 2003.

Imaz C. (2007) Los Clubes de oriundos en México en Cecilia Imaz (coordinadora) ¿Invisibles? Migrantes internacionales en la escena política. FCPyS UNAM SITESA, México.

INEGI (2015). Instituto Nacional de Estadística y Encuesta Nacional de la Dinámica Demográfica (ENADID) 2009. Tabulados básicos. México: INEGI. Recuperado $\begin{array}{llllll}\text { el } & 21 & \text { de } & \text { septiembre } & \end{array}$ http://www3.inegi.org.mx/sistemas/tabuladosbasicos/tabdirecto.aspx?s=est\&c=33 617. 
Lanly, G. y M. Basila Valenzuela (2004), en Guillaume Lanly - M. Basila Valenzuela (Comps.), Clubes de migrantes oriundos en los Estados Unidos, Universidad de Guadalajara, Guadalajara.

Lizárraga, A. (2005). Nos llevó la ventolera... El proceso de la Emigración rural al extranjero. Universidad Autónoma de Sinaloa, Sinaloa.

Moctezuma, M. (2003). "Territorialidad de los Clubes de Zacatecas en Estados Unidos" en Revista Migración y Desarrollo, núm. 1, octubre.

Montoya E. (2004) Montoya Zavala, Erika, Exportando trabajo. Importando Progreso. Migración mexicana a EU y remesas en Gabriel Leyva Solano, DIFOCUR y UAS.

Montoya E., Valenzuela, B. y García I.. (2008). Los sinaloenses en Phoenix Arizona, hegemonía poblacional, patrones migratorios e inserción laboral. En Memorias del ler Congreso Latinoamericano sobre Migración Internacional. Voces del Sur y Pre-Congreso ALAS.

Periódico El Debate de Culiacán, "Elota ingresa a la Cruzada Nacional contra el hambre", 13 de marzo 2014.

Pintor, R. (2011). "El habitus y los campos transnacionales en el proceso del transnacionalismo migrante" en Migraciones Internacionales, vol.6 no. 2 México jul./dic.

Pintor, R. (2015). El otro Agua Verde, Procesos transnacionales migrantes, UAS, Sinaloa.

Pintor, R. e I. García (2008). "Inversiones de los Clubes de migrantes Sinaloenses en Estados Unidos, realizadas en sus lugares de origen" en Revista Sinaloa: Causa Común, No. 34, abril.

Pintor, R y E. Sánchez (2012). "Repensar a Sinaloa como Estado emergente de la migración mexicana, Ánfora, Vol. 19, No. 32.

Revista de la Fraternidad Sinaloense en California (FSC) (1999). Pasadena, California, Abril.

Revista de la Fraternidad Sinaloense en California (FSC) (2000). Pasadena, California, Agosto.

Revista de la Fraternidad Sinaloense en California (FSC) (2008). Pasadena, California, Edición especial, informe de labores, Abril.

Sánchez, E. (2014). "Movilidad acotada y vulnerabilidad laboral en trayectorias laborales de migrantes sinaloenses en los ángeles california (2007-2012)" en Ra Ximhai, Vol 10, Núm. 1

Sánchez E., García, I. y Pintor, R. (2015). "Revisión crítica de los programas de coinversión entre comunidades migrantes transnacionales y los gobiernos federal y locales en México" en IDENTIDADES, num. 9 año 5. Diciembre. Pp. 25-42.

Secretaría de Desarrollo Social (2014), Informe de labores del 3x1 en Sinaloa. Documento interno, Sinaloa.

S, J. (2013). Espacio Económico y desarrollo regional en Sinaloa, Colección Geografía y Desarrollo, UAS- JP-Editores, Sinaloa.

Torres, A. (2001). "Remittances for Small-Scale Infrastructure and Small Enterprise Development in Mexico. Evidence from the Public - Private Infrastructure Advisory Facility Study", Approaches to Increasing the Productive Value of Remittances, IAF, ECLAC and World Bank Conference, 2001, Washington, D.C., 19 March. 
Valenzuela, B. (2006). "El papel de las organizaciones de migrantes en la definición de acciones y políticas públicas estatales hacia la migración a los Estados Unidos" en Rafael Fernández de Castro (Cords.) Políticas Migratorias en los Estados de México, ITAM-México

Villegas, F., E. (2014). "Factores que contribuyen o limitan el envío de remesas colectivas en Sinaloa" en Brianda E. Peraza Noriega (Cord.) Migración, Desarrollo regional y Cultura en Sinaloa. Sinaloa, UAS. 